\title{
La necesidad de un Pacto de Estado por la Ciencia en España
}

Los Jefes de Estado y de Gobierno de la Unión Europea (UE) reunidos en la cumbre de Lisboa del año 2000 textualmente afirmaron que Europa debía convertirse antes del 2010 en la economía basada en el conocimiento más competitiva y dinámica del mundo, si no quería perder su actual posición de privilegio. Posteriormente en la cumbre de Barcelona de 2002 profundizaron en este aspecto y decidieron que a final de la década el porcentaje del PIB dedicado a I+D debería alcanzar el 3\%.

España ha dado un gran salto adelante en los últimos 25 años. Nuestra calidad de vida y nuestro bienestar económicos parecen asegurados. Sin embargo, estamos viendo cómo la producción industrial, incluso la de productos complejos, se puede trasladar de un día para otro a los países del Este, donde los beneficios son mayores. La prensa da cuenta de nuevos casos cada semana. Si este goteo se convierte en un torrente, ¿de dónde saldrán los futuros puestos de trabajo? Ya empezamos a ver que la fabricación rutinaria no es la respuesta, los servicios tienen un alcance limitado, la agricultura necesita cada vez menos trabajadores. Así que la pregunta es ¿"qué haremos"? La respuesta de la cumbre de Lisboa es la correcta. Nos hemos de basar en la fuerza que actualmente nos diferencia de las economías emergentes del planeta, lo que se conoce como "Economía basada en el conocimiento". No hay alternativa.

Sin embargo, no se ven las acciones que deben llevar a hacer realidad los acuerdos de las cumbres de Lisboa y Barcelona. Si hemos de depender de una economía basada en el conocimiento, necesariamente hemos de generar más conocimiento que nos permita identificar nuevas áreas susceptibles de ser desarrolladas y convertidas en empresas rentables. Ahora bien, la creación de conocimiento no ocurre sin estímulos. Requiere recursos e inversión. Las cifras sin embargo no son prometedoras. España dedica un porcentaje alrededor del $1 \%$ a I+D y aún ahí hay camufladas muchas partidas que nada tienen que ver con la auténtica I+D. En cualquier caso, el porcentaje es irrisorio comparado con los países avanzados. Es- tamos condenados a perder la carrera si no aumentamos el nivel de inversión para estimular la actividad de investigación.

Existe el peligro de creer que dicha dinamización se alcanzará sólo con las inversiones empresariales. Eso es un error pues esta solución no puede engendrar por sí sola el dinamismo necesario. Las empresas se van a aquellos países que saben maridar la investigación pública (universitaria y Organismos Públicos de Investigación) de alto nivel con la investigación privada. Y ese lugar, hoy por hoy, son los Estados Unidos de América. Así estamos viendo cómo importantes laboratorios farmacéuticos están concentrando sus centros de investigación y desarrollo en América y cierran sus laboratorios en Europa.

El tema es de la mayor trascendencia. Por eso la comunidad centífica española ha lanzado la iniciativa de un Pacto de Estado por la Ciencia que nos permita eludir el riesgo de hipotecar nuestro crecimiento y progreso social en los próximos años. Su concreción requerirá una movilización sin precedentes. En dicho pacto deberían participar los Gobiernos central y autonómicos, partidos políticos, sindicatos y patronales, universidades y organismos de investigación, fundaciones, bancos y cajas de ahorro, con la complicidad de toda la sociedad. Por otra parte, el Pacto exige una convicción generalizada sobre el valor social del desarrollo científico. Es por tanto necesario un gran esfuerzo por parte de los científicos para que la sociedad en general sea consciente del valor de la ciencia y del impacto que tiene en el bienestar de todos y cada uno.

Dicho Pacto, cuyo texto completo puede encontrarse en las páginas siguientes, debe permitir que España progrese hacia esa economía basada en el conocimiento que determinará el nivel de vida de los países en el siglo XXI.
Joan J. Guinovart Director del IRBB-PCB, UB Barcelona 


\section{PACTO DE ESTADO POR LA CIENCIA}

En los últimos dos decenios el mundo desarrollado ha iniciado una profunda transformación. De la mano del conocimiento acumulado en áreas tan dispares como la ingeniería genética, la biología molecular, la física o las telecomunicaciones, entre muchas otras, el mundo que hoy conocemos ha pisado el acelerador rumbo al futuro. La biomedicina, la biotecnología y la nanotecnología, además de las potentes herramientas en genómica y proteómica, son sólo algunos de los ejemplos en los que se fundamenta este prodigioso cambio de paradigma.

España, hasta ahora, apenas ha contribuido a ninguno de los grandes conceptos y tecnologías con los que se está construyendo el futuro. Y ello a pesar de que el conocimiento acumulado en todas estas áreas y las aplicaciones que de ellas se están derivando marcan el camino a seguir en los terrenos de influencia económica, política y social. El papel del conocimiento científico como motor de competitividad, bienestar y calidad de vida, y liderazgo internacional, es algo que ya nadie pone en duda.

El camino elegido por España en los últimos 25 años es, en cierto modo, paradójico. En este tiempo la economía española ha logrado ocupar un lugar destacado en el escenario internacional. Y lo ha conseguido sin necesidad de invertir en la generación de conocimiento, sino ofertando buenos servicios, en especial en turismo y ocio; manteniendo una política industrial económicamente competitiva; y, no debe olvidarse, aprovechando los fondos de cohesión europeos. Estos tres factores, entre otros, han permitido a España alcanzar un importante nivel de bienestar y mirar al futuro con cierto optimismo.

En el momento actual, sin embargo, el modelo sobre el que se sustenta la economía española empieza a dar síntomas de agotamiento. La deslocalización industrial, la influencia creciente de economías emergentes, o una cada vez mayor dependencia científica y tecnológica del exterior, son amenazas más que reales. Estos problemas no permiten más retraso. Ha llegado, por tanto, el momento ineludible de diseñar una estrategia a medio y largo plazos para que la economía española y el bienestar de sus ciudadanos mantengan o incluso incrementen las cotas alcanzadas hasta hoy. Dadas las tendencias que se dibujan en la escena mundial, tan sólo la generación de conocimiento puede asegurar este objetivo. Los hechos demuestran que los países que ejercen hoy un liderazgo económico, político y social son aquellos que, en su día, decidieron apostar claramente por la innovación surgida de la investigación científica en las más diversas ramas. La innovación se ha traducido en conceptos y, con el tiempo, en tecnologías e industrias capaces de marcar la pauta. Los países líderes supieron ver, y todavía lo entienden del mismo modo, que invertir en ciencia es invertir en futuro.
España tiene a su alcance participar de este futuro. Para ello debe abandonar el furgón de cola europeo en el que, según todos los indicadores, ha permanecido todo este tiempo. Algo que será posible no sólo si se incrementa la inversión global en ciencia y tecnología, sino también si esa apuesta presupuestaria es gestionada adecuadamente en el medio y el largo plazos. El talento y la capacidad existen, pero se requieren voluntad y la valentía políticas para hacerlo posible.

Los firmantes de este documento, científicos biomédicos en activo, creemos representar el sentir de buena parte de la comunidad científica. Conscientes del grave déficit que hay que superar y de los beneficios que el esfuerzo inversor en ciencia puede aportar a la sociedad española, proponemos un gran Pacto de Estado por la Ciencia en el que participen, además del gobierno y todos los partidos políticos, las distintas Comunidades Autónomas, los agentes económicos y sociales y los propios científicos.

El Pacto de Estado por la Ciencia debe tomar en consideración, para su debate y posterior plasmación en compromiso público, al menos los siguientes objetivos:

\section{COMPROMISO POR EL DESARROLLO}

\section{Invertir en ciencia es invertir en desarrollo}

El gobierno de España, así como los de las Comunidades Autónomas, los partidos políticos y agentes económicos y sociales, además del sector industrial y los propios científicos, tienen que asumir que invertir en ciencia es invertir en desarrollo, productividad y en nivel y calidad de vida. Ello sólo es posible con un cambio de mentalidad que permita la consecución de un modelo que dé estabilidad en el tiempo, en las formas y en la financiación necesaria para acometer un salto de calidad.

\section{Europa como objetivo}

Las cumbres de jefes de Estado de Lisboa y Barcelona, ratificadas ambas por España, fijaron entre sus grandes objetivos alcanzar en 2010 una inversión media en investigación, desarrollo e innovación correspondiente al 3\% del PIB. Si España quiere invertir en su futuro y formar parte de la Europa desarrollada, debe asumir este compromiso. El Gobierno debe exigirse el diseño y ejecución de planes específicos para cumplir estas expectativas, haciendo de éste uno de sus grandes objetivos de futuro.

\section{España puede y debe contribuir a la vanguardia del conocimiento}

El nivel de desarrollo económico y social alcanzado por España en los últimos 25 años no se corresponde con su grado de desarrollo científico y tecnológico. Sólo la producción de ciencia de calidad puede equili- 
brar los indicadores, hacer más competitiva una economía basada en el conocimiento y dar el salto cualitativo que precisa para situarse en la vanguardia de la economía mundial.

\section{COMPROMISO INSTITUCIONAL}

\section{Esfuerzo de organización}

La ciencia, para que dé los frutos apetecidos, debe estar organizada cuidadosa y certeramente. Esto no ocurre en España. Al menos no en la medida de lo que sería necesario. Los intentos llevados a cabo hasta la fecha han arrojado, salvo excepciones, unos pobres resultados. Existe una gran dispersión de organismos públicos de los que depende la investigación y apenas hay instrumentos, como sucede en países avanzados, que medien en su coordinación. El actual Ministerio de Ciencia y Tecnología no ha sabido, o no ha podido, ejercer bien esta función. Tampoco ha sabido, o no ha podido, impulsar las medidas legales que modernicen el sistema y lo doten de la suficiente flexibilidad. Resulta imperativo revisar las prioridades, funciones y responsabilidades de ese ministerio.

\section{Esfuerzo de coordinación}

La productividad científica española se concentra mayoritariamente en universidades y Organismos Públicos de Investigación (OPI). Pero también se investiga, aunque mucho menos de lo que sería deseable, en hospitales y en la industria. El Ministerio de Ciencia y Tecnología, debidamente coordinado con las Comunidades Autónomas, podría y debería asumir un papel de liderazgo, del mismo modo que lo ejercen las grandes agencias de investigación de los países más avanzados. El modelo actual, en el que la participación de las universidades y de los centros dependientes del Sistema Nacional de Salud español brilla por su ausencia, resulta poco creíble, además de poco adecuado e ineficaz.

\section{Esfuerzo de planificación y de continuidad}

Sólo un instrumento con funciones y responsabilidades bien definidas y dotado de la independencia suficiente, puede diseñar y ejecutar un Plan Nacional de Investigación, Desarrollo e Innovación que cumpla los objetivos de calidad, estabilidad, prioridades y proyección de futuro que precisa España. La ciencia, aunque necesariamente vinculada a objetivos políticos, económicos y estratégicos, debe servir al país y no a otros intereses. No puede inventarse año a año ni carecer de calendarios estables. Tampoco puede permitirse el lujo de sustituir la planificación a largo plazo por la ciencia de escaparate, de elevado coste y discutible eficacia para el sistema.

\section{COMPROMISO DE FINANCIACIÓN}

Basta con poco para salir de pobres

La inversión que dedica España a ciencia y tecnología es a todas luces insuficiente para participar en los grandes proyectos de ciencia internacionales, para potenciar una industria tecnológicamente avanzada e innovadora o para liderar proyectos de investigación con impacto clínico de envergadura. El esfuerzo inversor que debería hacer España para alcanzar en pocos años la actual media europea del 2\% del PIB, aunque en términos absolutos pueda parecer importante, no lo es en términos relativos ni está fuera del alcance de los gobiernos si hay voluntad política para ello. Participar del futuro representa muy poco para el global de los Presupuestos Generales del Estado.

\section{Voluntad de transparencia}

La dispersión de fuentes de financiación, y sobre todo la inclusión de la I+D militar en las partidas presupuestarias destinadas a investigación, impide conocer con certeza de cuánto dinero dispone la ciencia. Sin transparencia en las cuentas, es imposible diseñar un plan de financiación estable y sostenido que garantice el crecimiento del sistema hasta acercarlo a la media europea.

\section{Estabilidad presupuestaria}

Durante algo más de una década, el presupuesto destinado a investigación ha permanecido prácticamente estancado. El gran déficit acumulado obliga a incrementos sostenidos y estables en el tiempo hasta alcanzar la media europea en los principales indicadores mediante planes plurianuales que tengan la vista puesta en 2010. Asimismo, es imperativo que los presupuestos destinados a este capítulo se ejecuten en su totalidad, algo que no ha sucedido en los últimos ejercicios.

\section{COMPROMISO CON EL INVESTIGADOR}

\section{Los grupos de investigación}

El mayor activo de la ciencia son los grupos de investigación. Invertir en ellos, por tanto, es invertir en futuro. Su financiación, sin embargo, es a menudo irregular, insuficiente y fragmentada, lo que obliga a recurrir a diversas fuentes que, por lo general, están descoordinadas o son incompatibles. Desarrollar cualquier proyecto de envergadura capaz de generar innovación, y por tanto riqueza, resulta muy difícil en estas condiciones. Por otra parte, debe visualizarse el apoyo decidido a los grupos de investigación consolidados y la incorporación de grupos emergentes al sistema. 


\section{La carrera investigadora}

El científico es la base de todo sistema de investigación. En España, no obstante, los mecanismos de promoción de nuevos investigadores ofrecen muy limitadas expectativas de futuro. Prima la precariedad frente a la estabilidad. Ello obliga a definir un marco adecuado y coherente para el desarrollo de la carrera investigadora. Asimismo, es necesario seguir potenciando la incorporación de científicos al sistema hasta alcanzar la media europea en 2010 (8,3 investigadores por cada 10.000 ciudadanos frente a los 4,2 actuales) y asegurar que las incorporaciones contarán con los medios adecuados para desarrollar su trabajo en función de la plaza a ocupar, el centro que lo acoja y los proyectos a desarrollar. En cualquier caso, no basta con ofrecer plazas de investigador: cada plaza de nueva creación debe ir asociada a superficie de investigación, a necesidades de infraestructura científica y al valor cualitativo del proyecto en el que se vaya a integrar, de acuerdo, además, con un sistema estable de contratación.

\section{La cuantía de los proyectos}

La cuantía media de los proyectos de investigación debería duplicarse, cuando no triplicarse, con el objetivo de acercarse cada vez más a la media europea. Asimismo, debería establecerse un modelo de gestión eficaz para la evaluación y seguimiento de unos proyectos cuyo valor, en general, debería tender a su coste real. Esto es, incluir las necesidades de personal técnico e investigador, becarios, superficie de investigación y acceso a redes y servicios científicos, además de a plataformas tecnológicas. Las dotaciones actuales difícilmente hacen viables proyectos de suficiente calidad y envergadura.

\section{COMPROMISO CON LA ESTRUCTURA}

\section{Política de centros}

Los centros de investigación, sean OPI, universitarios o dependientes del Sistema Nacional de Salud o de fundaciones u organizaciones sin ánimo de lucro, deben adecuarse con urgencia a las necesidades que plantea la ciencia actual. Salvo excepciones, España está falta de grandes centros con capacidad para competir internacionalmente en condiciones, y ninguna de nuestras universidades forma parte de las 100 mejores del mundo en resultados de investigación. Como en los países más avanzados, la política de centros debe visualizarse en forma de pirámide en cuya cúspide haya un grupo de referencia que se nutra y apoye en una base que ejerza el papel de cantera. La ciencia de calidad no es posible sin una buena y amplia base.

\section{Política de plataformas}

La ciencia actual exige servicios científico-técnicos, plataformas tecnológicas y el acceso a redes internacionales como condición para obtener resultados de calidad. Esto es especialmente cierto en áreas de alto valor estratégico como la biotecnología o la biomedicina, en las que la necesidad de plataformas como la genómica y la proteómica, o aquellas basadas en el conocimiento del gen, resultan fundamentales. España acumula un enorme retraso en este terreno. Y ello no sólo impide la obtención de resultados de calidad, sino incluso la simple participación en los grandes proyectos internacionales.

\section{Política industrial}

La vertebración de la buena ciencia con la buena industria genera riqueza y potencial económico. En España, salvo excepciones, apenas hay industrias que inviertan en investigación. El desarrollo de un plan consensuado por todos para tratar de involucrar a las empresas en el proceso de investigación resulta, en estos momentos, una exigencia prioritaria. También lo es la definición de instrumentos y mecanismos que potencien la transferencia tecnológica, garanticen la propiedad intelectual, la formación de empresas de base tecnológica, el acceso a fórmulas de financiación adecuadas y la incorporación de investigadores al tejido empresarial. Ello debe redundar en el apoyo formal a la industria realmente innovadora y tecnológicamente avanzada, especialmente en sectores de interés estratégico, y en el fomento de una mayor y más eficaz colaboración entre los sectores público y privado para el establecimiento de formas de cofinanciación de centros de investigación.

\section{Política traslacional}

Muchas ramas de la ciencia tienen aplicaciones evidentes. La biotecnología o la biomedicina son buenos ejemplos de ello. La primera, por su clara influencia sobre el desarrollo tecnológico y económico. La segunda, además, por su contribución a la mejora de la salud y la asistencia médica. España atesora un gran talento y potencial biomédicos. Los avances en biología molecular, genética y genómica, van a tener un gran impacto en la comprensión de la enfermedad y en el desarrollo de estrategias y procedimientos preventivos, diagnósticos y terapéuticos. Sin embargo, los mecanismos de transferencia de los laboratorios y unidades experimentales biomédicos a los sistemas de salud están poco desarrollados en nuestro país. Es esencial realizar un esfuerzo para saltar esa brecha. Parte de ese esfuerzo debería centrarse en consolidar unidades o centros de investigación en los grandes hospitales. Bastaría inicialmente con dotar a 20 o 30 
grandes hospitales con centros de investigación para dar un gran salto de producción, calidad y transferencia al ámbito de la salud. Sólo así se podría trasladar eficazmente a médicos y pacientes los beneficios de la investigación en ingeniería genética y biología molecular y celular de los últimos 25 años.

\section{COMPROMISO CON LA SOCIEDAD}

\section{Fuente de riqueza y bienestar}

Ciencia es sinónimo de progreso. Los países que han invertido en generación de conocimiento han logrado mejoras económicas y aumentado el nivel de bienestar de sus ciudadanos a un coste razonable. Los que no lo han hecho, han debido adquirir las mejoras a

\section{Jesús Ávila}

Presidente de la SEBBM

Director del CBM "Severo Ochoa"

(CSIC-UAM). Madrid

\section{Miguel Beato \\ Director del CRG, Barcelona}

\section{Joan J. Guinovart}

Director del IRBB-PCB, UB

Barcelona

\section{José López Barneo}

Jefe del Servicio de Investigación

Hospital Virgen del Rocío, Sevilla

\section{Ginés Morata}

Profesor de Investigación del CSIC.

\section{Vicente Rubio}

Presidente electo de la SEBBM

Director del IBV (CSIC), Valencia elevados costes y seguir el dictado de los que ejercen el liderazgo. España puede y debe apostar por la primera vía. Optar por la segunda es, desde todos los puntos de vista, una irresponsabilidad inconcebible.

\section{Fuente de conocimiento y cultura}

Ciencia es también sinónimo de cultura. Los países cultos se miden no sólo por sus artistas, literatos, músicos o pintores. También lo hacen por el nivel de sus científicos, de personajes capaces de entrar por la puerta de la Historia. Sólo Santiago Ramón y Cajal y Severo Ochoa ocupan este escalafón en España. Un bagaje demasiado pobre para un país como el nuestro.

\author{
Mariano Barbacid \\ Directo del CNIO. Madrid \\ Félix Goñi \\ Director de la Unidad de Biofísica \\ CSIC/UPV-EHU, Bilbao
}

Carlos Martínez Alonso

Director del Centro de Inmunología

y Oncología, CNB, Madrid

Federico Mayor Menéndez

Ex-Director del CBM "Severo Ochoa" Madrid

\section{Margarita Salas}

Ex-Presidenta del Instituto de España Miembro de la RAC y de la RAE 